\title{
Tetraspanins: Novel Molecular Regulators of Gastric Cancer
}

\author{
Yue Deng ${ }^{1}$, Sicheng Cai ${ }^{1}$, Jian Shen ${ }^{2}$ and Huiming Peng ${ }^{1 *}$ \\ ${ }^{1}$ Department of Human Anatomy, School of Basic Medicine, Tongji Medical College, Huazhong University of Science and \\ Technology, Wuhan, China, ${ }^{2}$ Department of Pancreatic Surgery, Union Hospital, Tongji Medical College, Huazhong \\ University of Science and Technology, Wuhan, China
}

\section{OPEN ACCESS}

Edited by:

Jianjun Xie,

Shantou University, China

Reviewed by:

Peter Monk

The University of Sheffield,

United Kingdom

Prabhash Dadhich,

Cellf Bio LLC, United States

*Correspondence:

Huiming Peng

hmpeng2003@hust.edu.cn

Specialty section:

This article was submitted to

Gastrointestinal Cancers,

a section of the journal

Frontiers in Oncology

Received: 29 April 2021

Accepted: 07 June 2021

Published: 18 June 2021

Citation:

Deng Y, Cai S, Shen J and Peng H (2021) Tetraspanins: Novel Molecular Regulators of Gastric Cancer. Front. Oncol. 11:702510. doi: 10.3389/fonc.2021.702510
Gastric cancer is the fourth and fifth most common cancer worldwide in men and women, respectively. However, patients with an advanced stage of gastric cancer still have a poor prognosis and low overall survival rate. The tetraspanins belong to a protein superfamily with four hydrophobic transmembrane domains and 33 mammalian tetraspanins are ubiquitously distributed in various cells and tissues. They interact with other membrane proteins to form tetraspanin-enriched microdomains and serve a variety of functions including cell adhesion, invasion, motility, cell fusion, virus infection, and signal transduction. In this review, we summarize multiple utilities of tetraspanins in the progression of gastric cancer and the underlying molecular mechanisms. In general, the expression of TSPAN8, CD151, TSPAN1, and TSPAN4 is increased in gastric cancer tissues and enhance the proliferation and invasion of gastric cancer cells, while CD81, CD82, TSPAN5, TSPAN9, and TSPAN21 are downregulated and suppress gastric cancer cell growth. In terms of cell motility regulation, CD9, CD63 and CD82 are metastasis suppressors and the expression level is inversely associated with lymph node metastasis. We also review the clinicopathological significance of tetraspanins in gastric cancer including therapeutic targets, the development of drug resistance and prognosis prediction. Finally, we discuss the potential clinical value and current limitations of tetraspanins in gastric cancer treatments, and provide some guidance for future research.

Keywords: tetraspanins, gastric cancer, tumor proliferation, tumor invasion, tumor metastasis, targeted therapy, drug resistance

\footnotetext{
Abbreviations: GC, gastric cancer; TM, transmembrane; N, amino; C, carboxyl; ECL, extracellular loop; TEMs, tetraspaninenriched microdomains; HCC, hepatocellular carcinoma; GPVI, glycoprotein VI; MMP-9, matrix metalloproteinase-9; uPA, urokinase plasminogen activator; MRP-1, motility-related protein; CAFs, cancer-associated fibroblasts; GIA, gastrointestinal adenocarcinoma; MAPK, mitogen-activated protein kinase; JNK/SAPK, c-Jun N-terminal kinase/stress-activated protein kinase; MDR, multidrug resistance; NOTCH2, Notch Receptor 2; 5-FU, 5-Fluorouracil; PIK3R3, Phosphoinositide-3-Kinase Regulatory Subunit 3; mAb, monoclonal Antibody.
} 


\section{INTRODUCTION}

Gastric cancer (GC) is the fourth most common cancer worldwide in men following lung, prostate, colorectal, and the fifth in women following breast, colorectal, cervical, lung. Risk factors for gastric cancer include Helicobacter pylori infection, age, high salt intake, and low-fruit and vegetables diets (1). About $70 \%$ of gastric cancer cases worldwide are in developing countries, including Eastern Asia, Central and Eastern Europe, and South America (2). The regional distribution variations suggest that the occurrence of gastric cancer is related to environmental factors and lifestyles (3). Patients with advanced gastric cancer usually start with a platinum and fluoropyrimidine doublet in the first line, and are treated with sequential lines of chemotherapy. Despite advances in treatment strategies recently, advanced gastric cancer patients still have a poor prognosis and the median survival is less than 1 year (1). Therefore, exploring the internal molecular mechanisms underlying gastric cancer development is conducive to generating more effective therapeutic targets and bringing hope to patients.

The tetraspanins belong to a protein superfamily with some common structural features. They have four hydrophobic transmembrane domains (TM1-TM4), short intracellular amino(N) and carboxyl(C) tails, a small intracellular loop, a small extracellular loop (ECL1), and a large extracellular loop (ECL2) (4). ECL2 is subdivided into a highly conserved region and a variable region. The conserved region has been revealed to mediate homodimerization, while the variable region is related to specific interactions with other proteins. Compared with ECL2, little is known about the function of ECL1. Within the intracellular regions, palmitoylation sites of cysteine residues work for tetraspanin web assembly, and the C-terminal tail contributes to specific functional links to cytoskeletal or signaling proteins. Four TM domains are important in 'tetraspanin web' biosynthesis and assembly as probable sites of intra- and inter-molecular interactions (5).

Currently, 33 mammalian tetraspanins have been reported and they are ubiquitously distributed in various cells and tissues (6). Some tetraspanins are detected to be abundantly expressed in specific tissues. For example, TSPAN32, CD37, and CD53 are tissue enhanced in blood and lymphoid tissue. TSPAN9, TSPAN5, and TSPAN7 are enriched in brain. TSPAN1, TSPAN11, and TSPAN8 are widely distributed in the intestine. TSPAN6 is in the salivary gland, TSPAN33 is in the kidney, while TSPAN21 is abundant in the prostate and urinary bladder. Other tetraspanins are low tissue specificity and are distributed in almost all tissues (7). On the cell membrane, tetraspanins interact with other membrane proteins to form tetraspanin-enriched microdomains (TEMs) and serve a variety of functions including cell adhesion, invasion, motility, cell fusion, virus infection, and signal transduction $(8,9)$. With a thorough study of tetraspanins, its role in multiple tumor development stages has been gradually revealed in recent years, such as early carcinogenesis, angiogenesis, proliferation, invasion, and metastasis (10). Accumulating studies found that tetraspanins play critical roles in gastric cancer development. Here, we review the current evidences on the function of tetraspanins in gastric cancer development and progression to provide some guidance for clinical treatment and future research.

\section{ROLE OF TETRASPANINS IN GASTRIC CANCER CELL GROWTH}

Tetraspanins have been confirmed to play an essential role in tumorigenesis and progression (10). Different tetraspanins contribute to diverse biological functions across cancer cells. Here, we summarize tetraspanins that enhance the proliferation and invasion of gastric cancer cells, including TSPAN8, CD151, TSPAN1, and TSPAN4 (Figure 1). We also discuss several tetraspanins, including CD81, CD82, TSPAN5, TSPAN9, and TSPAN21 that suppress gastric cancer cell growth (Figure 2).

\section{Tetraspanins That Facilitate Gastric Cancer Cell Proliferation and Invasion TSPAN8}

TSPAN8, also known as CO-029 or TM4SF3, belongs to the tetraspanin family and has been reported to be associated with multiple cancer types, such as hepatocellular carcinoma (11), pancreatic adenocarcinoma (12), colon carcinoma (13), breast cancer (14). TSPAN8 expression in tumor cells is related to increased metastasis $(10,15)$, proliferation (16), induction of angiogenesis (17) and thrombosis (18). The mechanism by which TSPAN8 has emerged as a key molecular is attributed to its position in TEMs and is primarily related to integrins, proteases, and cytoplasmic signaling molecules (19). Besides, the effect of TSPAN8 on angiogenesis may be partially mediated by exosomes (20).

As for gastric cancer, several studies have revealed that TSPAN8 expression is increased in gastric cancer tissues compared to normal tissues. Matsumura et al. found TSPAN8 was up-regulated in gastric cancer using microarray analysis (21). Mottaghi-Dastjerdi et al. performed suppression subtractive hybridization (SSH) on gastric adenocarcinoma tissue and the corresponding normal gastric tissue, and found TSPAN8 was overexpressed in the tumor (22). These findings suggest that overexpressed TSPAN8 may be related to the occurrence and progression of gastric cancer.

Further, ZHU's lab showed TSPAN8 acts as an oncogene in gastric cancer and promotes gastric cancer cell proliferation and invasion partially through EGFR signaling (23). The authors demonstrated that the expression of TSPAN8 was affected by EGF in a concentration- and time-dependent manner by in vitro experiments. When TSPAN8 was knocked down, the effect of EGF on promoting gastric cancer cell proliferation and invasion was attenuated.

Later in 2015, Wei et al. reported that TSPAN8 promotes gastric cancer cell proliferation and growth partially by activating the ERK MAPK pathway (24). Through MTT and transwellmatrigel assay, the authors found that TSPAN8 overexpression promotes the cell survival and invasion while TSPAN8 silencing has the opposite effect. They also found the expression of phospho-MEK1/2 and phospho-ERK1/2 was increased 


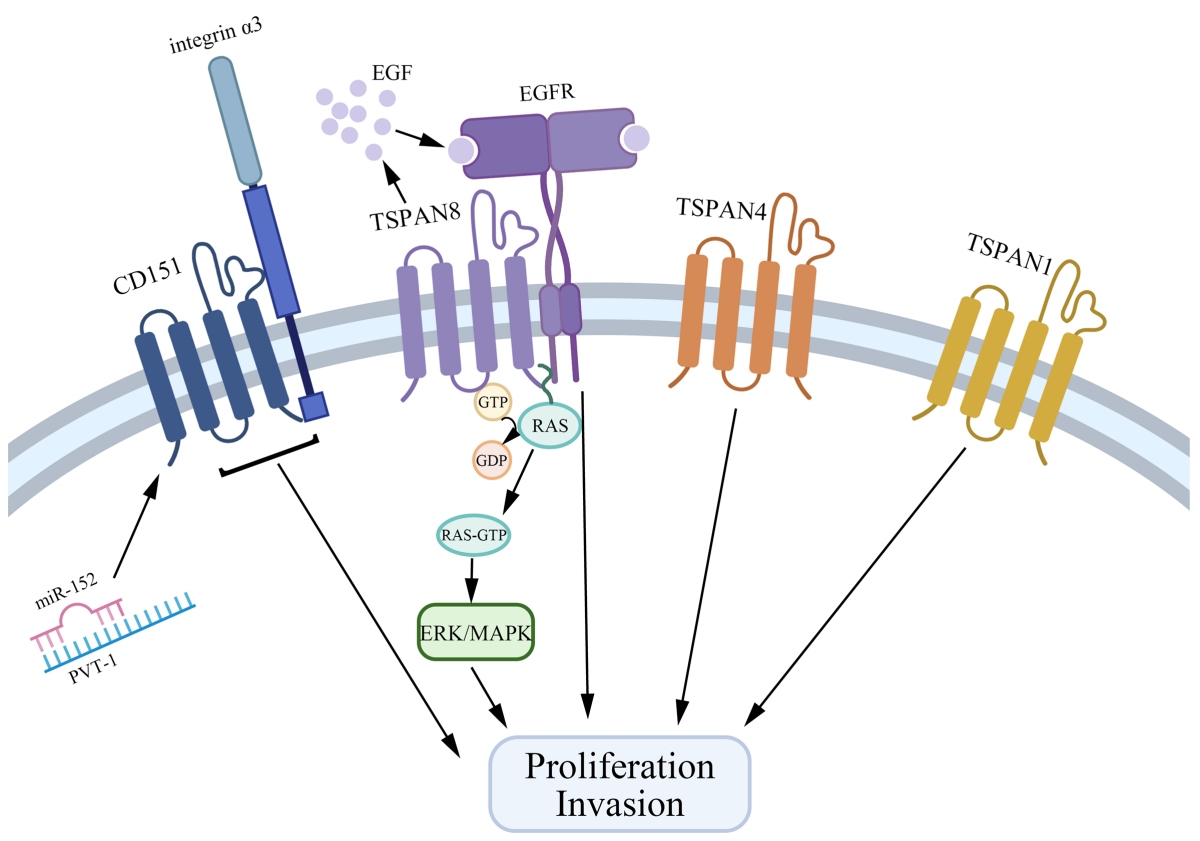

FIGURE 1 | Tetraspanins that promote gastric cancer cell proliferation and invasion. CD151, TSPAN8, TSPAN4, and TSPAN1 interact with other biomolecules in TEMs to facilitate the growth and invasion of gastric cancer cells. Especially, CD151 forms a complex with integrin $\alpha 3$, and on the other hand, PVT1 could bind to miR-152 to inhibit the expression of miR-152 to promote gastric cancer cell growth. TSPAN8 regulates gastric cancer cell proliferation via mediating the effect of EGF and activating the ERK MAPK pathway.

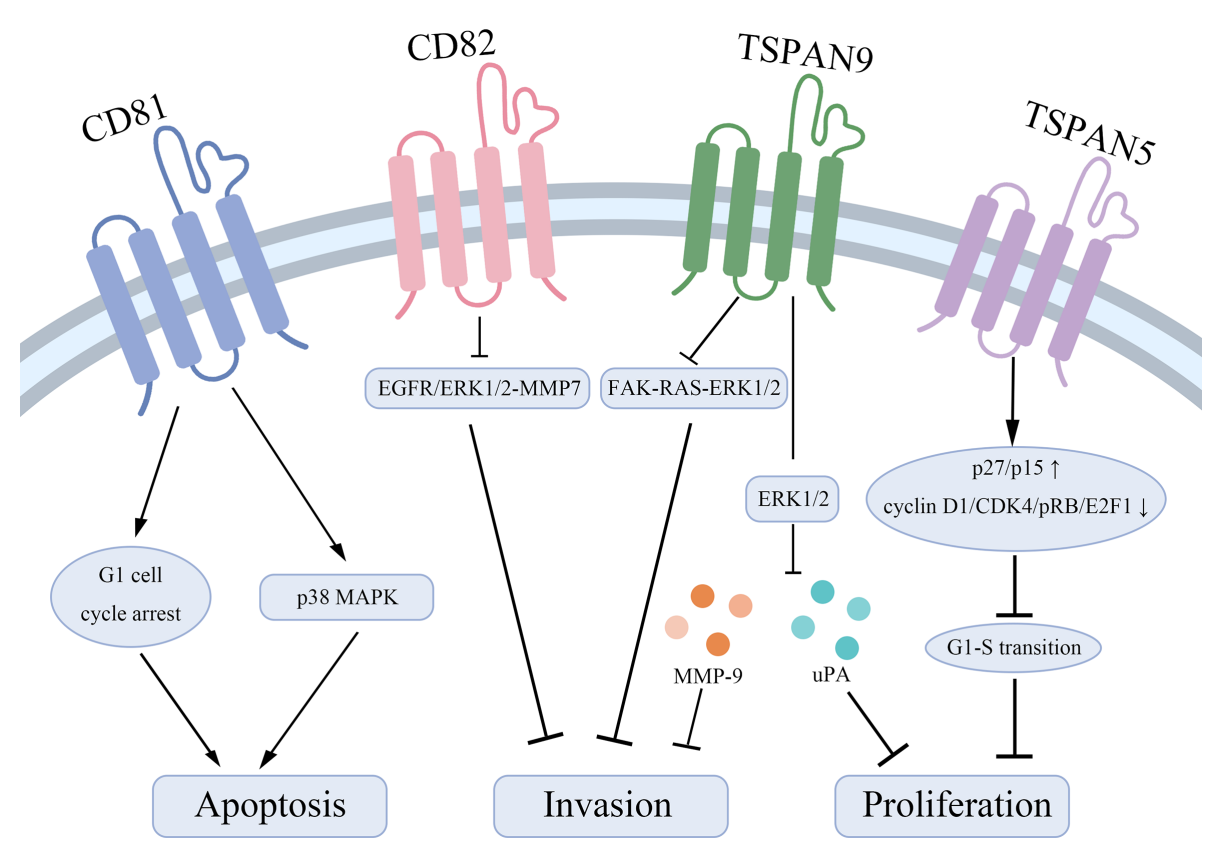

FIGURE 2 | Tetraspanins that suppress gastric cancer cell proliferation and lead to apoptosis. CD81 acts as a pro-apoptotic effector through inducing a G1 cell cycle arrest and inhibiting the phosphorylation of p38 MAPK. CD82 suppresses the EGFR/ERK1/2-MMP7 signaling pathway to represses gastric cancer invasion. TSPAN9 inhibits the ERK1/2 pathway to downregulate the expression of MMP-9 and UPA and inhibits the FAK-RAS-ERK $1 / 2$ signal pathway to repress invasion of gastric cancer cells. TSPAN5 suppresses the tumor proliferation via increasing the expression of p27/p15 and decreasing the expression of cyclin D1, CDK4, pRB, and E2F1 to control cell cycle transition. 
dramatically in the TSPAN8 overexpression cells but decreased in the TSPAN8 suppressed cells. When MER-ERK was inhibited in TSPAN8 overexpression cells, the increased survival rate and migrated cell number caused by TSPAN8 overexpression were significantly reduced. Therefore, the research by Wei et al. suggested that the MAPK pathway was involved in the effects of TSPAN8 on gastric cancer cell proliferation and invasion.

Recently, a novel study indicated a negative relationship between the expression of TSPAN8 and miR-324-5p in gastric cancer cells. MiR-324-5p was demonstrated to repress the viability and induce the apoptosis of gastric cancer cells via down-regulating TSPAN8. They also proposed that the possible mechanism was the combination of TSPAN8 3'UTR and miR324-5p (25). However, there are still few milestones on the treatment of gastric cancer targeting the above mechanisms.

\section{CD151}

CD151 has a broad distribution in the endothelium, epithelium, Schwann cells, and dendritic cells, as well as in skeletal, smooth, and cardiac muscle (26). It directly or indirectly interacts with abundant other transmembrane proteins to form TEMs and regulates integrin-dependent adhesion strengthening, cell morphology, and cell migration as a spectacular partner of laminin-binding integrins $(8,27)$. Karamatic Crew et al. revealed that CD151 was crucial for the proper assembly of the glomerular and tubular basement membrane in the kidney. In the skin, the inner ears, and erythropoiesis, CD151 also had functional significance. Therefore, it is not surprising that CD151 mutation is associated with end-stage kidney failure (28). As a major partner of laminin-binding integrins, CD151 modulates cancer cell motility, invasion, and metastasis together with $\alpha 3 \beta 1$ and $\alpha 6 \beta 4$ (15). For example, in hepatocellular carcinoma (HCC), CD151 was overexpressed compared with normal liver tissues and the expression level was positively related to the metastatic potential of HCC cell lines (29).

Evidences implicate that CD151 forms a complex with integrin $\alpha 3$ in gastric cancer cells and is positively associated with the invasiveness of gastric cancer (30). In 2014, Zhai et al. demonstrated in gastric cancer, miR-152 was downregulated and overexpressed miR-152 inhibited the proliferation and motility of gastric cancer cells via targeting CD151 (31). Later, Li et al. found PVT1, a long noncoding RNA, highly expressed in human gastric cancer tissues and correlated with lymph node invasion of gastric cancer (32). PVT1 could increase the expression of CD151 through binding to miR-152 and inhibiting the expression of miR-152 to promote gastric cancer (33). The authors likened PVT1 to a "sponge" in gastric cancer to inhibit miR-152, and made it an emerging potential therapeutic target for gastric cancer (33).

\section{Other Tetraspanins \\ TSPAN1}

TSPAN1 (NET-1) is identified to express in epithelial cell lines and multiple tumor cell lines including cervical carcinoma, lung carcinoma, squamous carcinoma, colon carcinoma, and breast carcinoma (34). In gastric cancer, Chen et al. elaborated the clinicopathological significance of overexpressed TSPAN1.
They found the expression level of TSPAN1 was positively related to the clinical stage and lymph node status of the tumor, while negatively associated with cancer cell differentiation and survival rates (35). Later, Lu et al. detected that the expression of TSPAN1 was dramatically increased in gastric cancer tissues, and clarified TSPAN1 as an oncogene to promote gastric cancer cell proliferation and invasion. Moreover, they identified that overexpressed miR-573 inhibited growth and invasion, induced G1/G0 arrest of gastric cancer cells through directly targeting 3'UTR of TSPAN1. This miR-573/TSPAN1 axis provides a novel perspective on the molecular mechanisms of gastric cancer (36).

\section{TSPAN4}

The role of TSPAN4 in gastric cancer was discovered through bioinformatics analysis. TSPAN4 was identified as one of the upregulated differentially expressed genes and the increased expression indicated a decreased survival rate. Moreover, the downregulation of TSPAN4 remarkably reduced the proliferation of gastric cancer cells (37). Therefore, TSPAN4 may be a biomarker and a potential therapeutic target for gastric cancer.

Together, TSPAN8, CD151, TSPAN1, and TSPAN4 are overexpressed in gastric cancer tissues and are related to a higher clinical stage and poorer prognosis via interacting with other molecules in TEMs. Specifically, TSPAN8 mediates the effect of EGF and actives the ERK MAPK pathway to promote gastric cancer cell proliferation. CD151 exerts its action by forming a complex with integrin $\alpha 3$. Also, many microRNAs are reported to be bound with the expression of tetraspanins, which provides a new idea for gastric cancer therapy.

\section{Tetraspanins That Repress Gastric Cancer Cell Proliferation CD81}

CD81 (TAPA-1), whose gene has been mapped to chromosomal region $11 \mathrm{p} 15.5$, is discovered as the target of an antiproliferative antibody initially $(38,39)$. As a protein widely distributed on the surface of various cell membranes, CD81 has been revealed to affect morphology, adhesion, activation, proliferation, and differentiation of B, T and other cells (40). On the surface of B cells, CD81 forms a complex with CD21, CD19, and Leu13. The complex reduces the signal transduction threshold for activating B cells mediated by B cell receptors (40,41). Similarly, CD81 interacting with $\mathrm{CD} 4$ and $\mathrm{CD} 8$ on $\mathrm{T}$ cells provides CD3 a costimulatory signal (42). In nonimmune cells, CD81 assists in egg fusion with sperm (43), myoblasts fusion during muscle regeneration (44) and exerts as a cell surface receptor for hepatitis C virus entry into the cell (45). In human lymphomas, CD81 expresses differentially, with increased expression in diffuse large B-cell lymphomas, but decreased expression in multiple myeloma, Hodgkin lymphoma, myeloid leukemia, and leukemic blasts of precursor B-cell lymphoblastic leukemia $(46,47)$.

However, in gastric cancer, CD81 is assessed as a tumor suppressor gene and CD81 downregulation is related to the malignant progression of the tumors (48). Yoo et al. proposed that the decreased expression of CD81 mRNA was due to 
aberrant $\mathrm{CpG}$ hypermethylation of its promoter but rarely due to genetic alterations. This downregulation facilitates the G1 to S transition of the cell cycle, while increased CD81 expression induces a G1 cell cycle arrest and promotes apoptosis. Moreover, downregulated CD81 significantly attenuates cellular responses to a variety of apoptotic stress signals, such as etoposide, 5-FU, doxorubicin, $\gamma$-irradiation, and hypoxia. Also, CD81 decreases the colony-forming ability of gastric tumor cells and inhibits the phosphorylation of p38 MAPK. Therefore, CD81 has antiproliferative and pro-apoptotic functions in gastric cancer cells and acts as a tumor suppressor gene.

\section{Other Tetraspanins \\ CD82}

As a metastasis suppressor gene, CD82 is also closely related to the gastric tumor cell invasion and metastasis. Xu's lab disclosed that CD82 downregulates the expression of phosphorylated(p)-EGFR, p-ERK1/2, and MMP7 to suppress the EGFR/ERK1/2-MMP7 signaling pathway. Therefore, CD82 inhibits the invasion of gastric cancer (49). Meanwhile, in gastric tumor cells, nuclear Drosha, an enzyme of endonuclease RNase III, promotes miR197 biosynthesis. The increased miR-197 downregulates CD82 to activate EGFR-ERK1/2-MMP7 signaling pathway, thus having an effect on promoting gastric tumor cells invasion and metastasis.

\section{TSPAN5}

TSPAN5 (NET-4, TMS4SF9) is shown to be highly expressed in the neocortex, the hippocampus, the amygdala, and murine cerebellar Purkinje cells, suggesting that TSPAN5 is of importance in the maintenance of brain activity in mice (50). It is also reported that TSPAN5 contributes to osteoclast formation and differentiation (51). In gastric cancer, the expression of TSPAN5 is significantly reduced and inversely correlated with tumor size and TNM stage, which indicates that TSPAN5 works as a tumor suppressor to inhibit the tumor proliferation, colony formation, and migration. Further, TSPAN5 increases the expression of p27/p15 and decreases the expression of cyclin D1, CDK4, pRB and E2F1, especially cyclin D1/CDK4, to control cell cycle transition from G1-S phase (52).

\section{TSPAN9}

TSPAN9 (NET-5, PP1057) is elucidated to regulate the platelet function through synergy with the collagen receptor GPVI (glycoprotein VI) and integrin $\alpha 6 \beta 1$ (53). Li et al. reported that overexpressed TSPAN9 inhibited the proliferation, migration, and invasion of human gastric cancer SGC7901 cells. TSPAN9 suppresses the ERK1/2 pathway to downregulate the proteins associated with tumor metastasis including matrix metalloproteinase-9 (MMP-9) and urokinase plasminogen activator (uPA) (54). Recently, Qi et al. found TSPAN9 inhibited migration and invasion of gastric cancer cells via inhibiting the FAK-RAS-ERK1/2 signal pathway. Furthermore, they confirmed EMILIN1, an extracellular secretory protein, exerted an anti-tumor effect by increasing the expression of TSPAN9 (55).

\section{TSPAN21}

TSPAN21 (UPK1A) is highly specifically expressed in normal urothelium, and can be observed in normal genitourinary tract, uterus and prostate (4). Kar et al. found TSPAN21 inhibited the down-regulation of MMP7 to regulate cell metastasis, invasion and survival. Loss the expression of TSPAN21 can lead to cell proliferation, metastasis and invasion (56). In gastric cancer, Zheng et al. reported that the protein level of TSPAN21 was significantly reduced, and the low expression of TSPAN21 was related to the poor prognosis of gastric cancer. When TSPAN21 was overexpressed, the invasion and migration of gastric cancer cell lines was inhibited (57). This indicates that TSPAN21 has a potential tumor suppressor effect in gastric cancer, but the mechanism remains to be fully explored.

Taken together, CD81, CD82, TSPAN5, TSPAN9, and TSPAN21 are regarded as tumor suppressors in gastric cancer to inhibit tumor cell growth and invasion, and enhance the sensitivity to apoptotic stress signals. Mechanically, CD82 represses the EGFR/ERK1/2-MMP7 signaling pathway and TSPAN9 suppresses the ERK1/2 pathway and the FAK-RASERK1/2 signal pathway to play biological roles.

\section{ROLE OF TETRASPANINS IN GASTRIC CANCER CELL METASTASIS}

Tetraspanins regulate cell motility, adhesion, and migration by interacting with integrins, signal molecules and other transmembrane proteins in TEMs. However, different tetraspanins can achieve even totally opposing functions in cancer cell metastasis. Here, we focus on the insights into the roles and molecular mechanisms of three tetraspanins involved in gastric cancer cell metastasis, CD9, CD63, and CD82 (also known as KAI1).

\section{CD9 and CD63}

CD9 was initially identified as a $24-\mathrm{kDa}$ surface protein specific for acute lymphoblastic leukemic cells. However, CD9 is also widely expressed on normal platelets and several nonhematopoietic tissues such as fibroblasts $(58,59)$. Later in 1991, CD9 was identified as a motility-related protein (MRP-1) to suppress motility and metastasis of multiple cancerous cell lines $(60,61)$. A significant feature of CD9 is that it tends to interact with various integrins including $\alpha 1 \beta 1, \alpha 2 \beta 1, \alpha 3 \beta 1$, $\alpha 4 \beta 1, \alpha 5 \beta 1, \alpha 6 \beta 1, \alpha 6 \beta 4, \alpha \operatorname{IIb} \beta 3$, and other transmembrane proteins including the EWI family, EGFR and DDR1 within TEMs $(62,63)$. Therefore, the potential of CD9 to regulate the motility is attributed to the association with these molecules.

CD63, mapped to chromosome region $12 \mathrm{p} 12 \rightarrow 12 \mathrm{q} 13$, was initially reported as an early stage-specific marker of melanoma progression because of the strong-expression in dysplastic nevi and radial growth phase primary melanoma (64). In histological studies, CD63 is related to melanoma malignancy and is differentially expressed in primary and metastatic lesions (65). However, another report has shown no significant difference in the expression of CD63 between primary and metastatic melanoma (66). Moreover, CD63 is involved in phagocytic and intracellular lysosome-phagosome fusion events (67).

Chen's lab found the expression level of CD9 and CD63 was decreased in gastric cancer. They proposed that CD9 protein level 
was inversely associated with lymph node metastasis and the reduction of CD9 was strongly associated with an increasing recurrence risk. Furthermore, the downregulation of CD63 also promotes metastasis and CD63 may serve as a marker for metastatic potential of gastric cancer (67). The mechanism of CD63 and CD9 on regulating motility is reported to be similar and both associate with $\beta 1$ and $\beta 3$ integrins (8).

However, in 2018, Miki et al. confirmed that CD9-positive exosomes from cancer-associated fibroblasts (CAFs) increased the migration and invasion abilities of scirrhous-type gastric cancer cells through activating MMP2 (68). And the prognosis of patients with positive CD9 in cancer and/or stromal cells was worse than the patients with dual CD9-negative expression. Their experiments revealed the unique role of CD9 in scirrhous-type gastric cancer.

\section{CD82/KAl1}

CD82 was originally discovered from $\mathrm{T}$ cell activation study in 1991 (69). In the same year, Ichikawa et al. found CD82 as a metastasis suppressor gene in prostatic cancer (70). Later in 1995, Dong, Isaacs, and Barrett isolated a metastasis suppressor gene from human chromosome $11 \mathrm{p} 11.2$ and designated it as KAI1 which is identical to CD82 (71). CD82/KAI1 associates with the proteins related to cell migration such as cell adhesion molecule, growth factor receptor, and signaling molecule in TEMs (72). Therefore, CD82 suppresses multiple metastasis stages, including cell motility and invasion, proliferation, apoptosis, and senescence (73). Moreover, CD82 promotes homotypic cell-cell adhesion, which plays an important role in suppressing metastasis. For example, overexpressed CD82 promotes E-cadherin-mediated intercellular adhesion in nonsmall cell lung carcinoma via stabilizing E-cadherin/ $\beta$-catenin complex formation (74). In various solid tumors, many studies have demonstrated that CD82 is a wide-spectrum invasionand metastasis-suppressor via regulating the functions of associated proteins, redistributing the plasma membrane components, post-translational modifications, and inducing apoptosis (72).

The metastasis suppression effect of CD82/KAI1 has also been confirmed in gastric cancer. As early as 1998, Hinoda et al. found CD82 expressed in normal fundic glands and intestinal metaplasia of the stomach but a decreased or lost expression in intestinal-type gastric cancer, especially the less differentiated type. They suggested an inversely relationship between CD82 expression and the progression of gastric cancer. However, whether CD82 is a metastasis suppressor gene in gastric cancer was not verified at that time (75). Later in 2007, decreased expression of CD82 in lymph node and liver metastases of gastric cancer compared with the primary tumors was shown by Yu's lab. Their studies indicated CD82 as a metastasis suppressor in gastric cancer and higher expression of CD82 reduced the metastatic potential (76).

In the same year, Zheng et al. obtained a similar conclusion that the expression of CD82 is negatively associated with liver metastasis of gastrointestinal adenocarcinoma (GIA) (77). However, Zheng's lab found that CD82 was expressed in the gastric hyperplastic gland and up-regulated in GIA, thereby proposing that CD82 was related to a physiological process in the gastrointestinal mucosa. And the overexpression was due to malignant transformation of mucosal epithelial cells or the upregulation of transcriptional regulators of CD82 (77).

In summary, the dominant view is that $\mathrm{CD} 9, \mathrm{CD} 63, \mathrm{CD} 82$ are metastasis suppressors and are negatively correlated with gastric cancer progression and lymph node metastasis. But interestingly, several recent studies suggest diverse perspectives in this regard. CD9-positive exosomes from CAFs increase the migration abilities of scirrhous-type gastric cancer cells and the prognosis is worse in patients with positive CD9 in cancer cells. In GIA, the expression level of CD82 is upregulated and this overexpression may be attributed to malignant transformation of mucosal epithelial cells. Although these studies are relatively superficial, we have a new understanding of tetraspanins, especially the role in gastric cancer metastasis.

\section{CLINICOPATHOLOGICAL SIGNIFICANCE OF TETRASPANINS IN GASTRIC CANCER}

\section{Therapeutics of Gastric Cancer That Target CD9}

As mentioned earlier, CD9 has an inhibitory effect on gastric cancer cell migration, and it plays a vital role in the development of gastric cancer, so CD9 may be a potential therapeutic target for gastric cancer. Nakamoto et al. revealed that ALB6, an anti-CD9 $\mathrm{mAb}$, significantly inhibited gastric cancer proliferation, angiogenesis, and promoted apoptosis in vivo in a mouse xenograft model of human gastric cancer (78). This anti-CD9 $\mathrm{mAb}$ ALB6 could be used to treat gastric cancer for the following reasons. First, the ligation of CD9 with ALB6 enhances the function of CD9 (79). Mechanically, ALB6 treatment-mediated apoptosis is achieved through activating the c-Jun $\mathrm{N}$-terminal kinase/stress-activated protein kinase (JNK/SAPK), p38 mitogen-activated protein kinase (MAPK) and Caspase- 3. However, ALB6 induced tyrosines phosphorylation of the p46 $\mathrm{Shc}$ isoform and the overexpression of its dominant-negative form inhibit ALB6-induced activation of JNK/SAPK, p38 MAPK, and Caspase-3, which leads to apoptosis suppression (80). Therefore, ALB6 can only limitedly activate p46 Shc isoform to induce apoptotic signals. Moreover, CD9 expression in gastric cancer is higher than non-cancerous tissues, thereby the adverse effects of anti-CD9 mAb therapy might be tolerable (81). In summary, CD9 maybe a powerful potential molecular target for gastric cancer therapy, but there is still a long way to go in improving the effectiveness of the treatment and overcoming the side effects.

\section{Tetraspanins Promote Gastric Cancer Drug Resistance TSPAN8}

A major obstacle in treating gastric cancer is the development of multidrug resistance (MDR) to chemotherapy in cancer cells (82). It is reported that MDR in tumor cells associates with 
several signaling pathways, including the $\mathrm{Wnt} / \beta$-catenin signal pathway in pancreatic cancer (83), the IL-6/STAT3/Jagged-1/ Notch axis in gastric cancer (84) and so on. TSPAN8 is a prodrug resistance protein in gastric cancer cells, while the silencing of TSPAN8 enhances the sensitivity of cancer cells to the cisplatin, 5-FU and adriamycin (85). TSPAN8 activates the Wnt/ $\beta$-catenin pathway via binding to NOTCH2 and increases $\beta$-catenin expression and accumulation in the nucleus to form MDR (85). Overall, TSPAN8 inhibitors may be developed as an adjuvant therapy of gastric cancer to reduce the resistance of cancer cells.

\section{TSPAN9}

5-Fluorouracil (5-FU) is a chemotherapeutic agent used for various malignant tumors, especially gastrointestinal cancers such as colorectal cancer, gastric adenocarcinoma and pancreatic cancer (86). However, the resistance to 5-FU has become a significant obstacle to the treatment of gastric cancer (87). Recently, Qi et al. demonstrated that 5-FU resistant gastric cancer cells had a high expression of TSPAN9 and TSPAN9 bound to PIK3R3 (p55) to suppress PI3K/Akt/mTOR pathway activation, which promoted autophagy and resulted in 5-FU resistance (88). Therefore, TSPAN9 inhibitors are shedding light for 5-FU-resistant gastric cancer patients.

\section{TSPAN20/UPK1B}

It is identified that UPK1B can be used as a biomarker to predict the chemotherapeutic outcomes of capecitabine and oxaliplatin in gastric cancer patients (89). The high expression of UPK1B in adjuvant capecitabine and oxaliplatin treated patients with GC was associated with poor outcomes. Some studies have shown that after knocking down UPK1B in cancer cells, the expression of key genes in the $\mathrm{Wnt} / \beta$-catenin signaling pathway is inhibited (90). Thus, it is speculated that UPK1B regulates oxaliplatin drug sensitivity through the $\mathrm{Wnt} / \beta$-catenin signaling pathway.

\section{Tetraspanins Predict the Prognosis of Gastric Cancer}

Tetraspanins have important significance in the occurrence, proliferation, invasion, and metastasis of gastric cancer. Different tetraspanins with increased or decreased expression level in cancer tissues can serve as the prognosis factor of gastric cancer (Table 1). The expression of TSPAN20/UPK1B (91), TSPAN1 (35), TSPAN8 (92), CD9 (93) and CD151 (30, 94-96) is positively associated with the clinical stage of gastric cancer and indicate a poor prognosis, while TSPAN5 (52), TSPAN21/UPK1A (57), CD82/KAI1 (97-100) are opposite. Especially, TSPAN9 expression is significantly decreased in gastric cancer tissues compared with the adjacent non-cancerous tissues but the high expression of TSPAN9 is associated with a poor prognosis (101). These tetraspanins, as biomarkers, have guiding significance in the diagnosis and prognosis prediction of gastric cancer. It is noteworthy that in previous studies, the overexpression of UPK1B mRNA is associated with laryngeal cancer recurrence (102), but Dai et al. found that UPK1B is negatively correlated with the prognosis of gastric cancer through bioinformatics analysis (91). This suggests that we can make full use of the database and data mining to further explore the functions of other tetraspanins in gastric cancer.

\section{CONCLUSIONS AND FUTURE PROSPECTS}

Tetraspanins interact with diverse other molecules and transmembrane proteins in TEMs, as well as in gastric cancer cells. Tetraspanin family can be seen in each stage of the occurrence and development of gastric cancer, from the growth, apoptosis, invasion and metastasis to the molecular targeted therapy and prognosis. Nonetheless, very little was found in the literature on the underlying molecular mechanisms of tetraspanins in gastric

TABLE 1 | Tetraspanins with prognosis prediction of gastric cancer

\begin{tabular}{|c|c|c|c|c|c|c|c|c|}
\hline \multicolumn{9}{|c|}{ Clinicopathological Factors } \\
\hline Tetraspanin & $\begin{array}{l}\text { Expression } \\
\text { level in GC }\end{array}$ & Tumor size & $\begin{array}{c}\text { Tumor } \\
\text { Differentiation }\end{array}$ & $\begin{array}{l}\text { Lymph node } \\
\text { Metastasis }\end{array}$ & TNM Stage & $\begin{array}{l}\text { Clinical Stage } \\
\text { (I/II and III/IV) }\end{array}$ & Survival Rate & Reference \\
\hline TSPAN20 & Upregulated & & & & & & Negative & (91) \\
\hline TSPAN1 & Upregulated & & Negative $\left.{ }^{\star \star \star}\right)$ & Positive $\left(^{\star \star \star}\right)$ & & $\begin{array}{l}\text { Positive } \\
\left({ }^{\star \star}\right)\end{array}$ & $\begin{array}{l}\text { Negative(within } 3 \text { years } \\
{ }^{\star *} \text {; within } 5 \text { years }{ }^{\star \star *} \text { ) }\end{array}$ & (35) \\
\hline TSPAN8 & Upregulated & & & & NS & NS & Negative $\left(^{\star \star \star}\right)$ & (92) \\
\hline CD9 & Upregulated & & NS & Positive $\left(^{\star \star \star}\right)$ & & $\begin{array}{l}\text { Positive } \\
\left({ }^{\star \star \star}\right)\end{array}$ & & (93) \\
\hline CD151 & Upregulated & $\begin{array}{l}\text { NS (94); } \\
\left.\text { Positive( }{ }^{\star \star}\right) \\
(30)\end{array}$ & $\begin{array}{l}\text { Negative }\left(^{\star \star}\right)(94) ; \\
\text { Negative }\left(^{\star}\right)(30)\end{array}$ & & $\begin{array}{l}\text { NS (95); } \\
\left.\text { Positive }{ }^{\star \star \star}\right) \\
(30)\end{array}$ & & Negative $\left(^{\star \star \star}\right)(30,94-96)$ & $(30,94-96)$ \\
\hline TSPAN5 & Downregulated & $\begin{array}{l}\text { Negative } \\
\left({ }^{\star \star \star}\right)\end{array}$ & NS & Negative $\left(^{\star \star}\right)$ & Negative $\left.{ }^{* \star *}\right)$ & & Positive $\left(^{\star \star \star}\right)$ & $(52)$ \\
\hline TSPAN21 & Downregulated & & Positive $\left(^{\star \star}\right)$ & Negative $\left(^{\star \star \star}\right)$ & Negative $\left(^{(\star \star}\right)$ & & Positive $\left(^{(\star)}\right)$ & $(57)$ \\
\hline CD82 & Downregulated & NS $(97,98)$ & $\begin{array}{l}\left.\text { Positive( }{ }^{\star \star \star}\right)(97) ; \\
\left.\text { Positive( }{ }^{\star}\right)(99)\end{array}$ & $\begin{array}{l}\text { Negative }\left(^{* \star}\right)(98) ; \\
\text { Negative }\left(^{\star \star \star}\right)(97,99)\end{array}$ & $\begin{array}{l}\left.\text { Negative }{ }^{\star \star \star}\right) \\
(97,98)\end{array}$ & Negative $\left(^{\star \star \star}\right)(99)$ & Positive $^{(* * *)}(98-100)$ & $(97-100)$ \\
\hline TSPAN9 & Downregulated & Positive $\left(^{\star \star}\right)$ & Negative $\left.{ }^{\star \star}\right)$ & Positive( $\left.{ }^{\star \star \star}\right)$ & Positive $\left(^{\star \star}\right)$ & & Negative $\left(^{\star \star \star}\right)$ & $(101)$ \\
\hline
\end{tabular}

${ }^{*} p<0.10 ;{ }^{* *} p<0.05 ;{ }^{* * *} p<0.01$; NS, Not Significant. Positive means a higher expression level of tetraspanins indicating a larger tumor size, higher tumour differentiation, more lymph node metastasis, more advanced TNM stage and clinical stage, a better survival rate. Negative is opposite. 
cancer, increasing the difficulty of its clinical application and targeted therapy. Thus far, the potential candidate therapeutic targets of tetraspanins in gastric cancer have mainly involved mAbs and mRNAs. Animal experiments have shown that ALB6, a mAb targeting CD9, can significantly inhibit the progression of gastric cancer $(79,80)$. The overexpression of some miRNAs also inhibits the proliferation and invasion of gastric cancer cell via targeting tetraspanins [for example, miR-324-5p and TSPAN8 (25), miR-152 and CD151 (31), miR-573 and TSPAN1 (36)]. Therefore, using these mAbs or upregulating the expression level of these miRNAs might be beneficial for the treatment of gastric cancer. Tetraspanins can also be used as therapeutic targets to overcome drug resistance or to increase drug sensitivity. However, research in clinical application is in its infancy, and there is still a long way to go before biological agents targeting tetraspanins are applied in clinical practice. Though the clinical researches of tetraspanins and gastric cancer are a drop in the bucket, we are still looking forward to more studies to reveal deep connection between tetraspanin family and gastric cancer, so as to find more potential and powerful therapeutic targets of gastric cancer.

\section{REFERENCES}

1. Smyth EC, Nilsson M, Grabsch HI, van Grieken NC, Lordick F. Gastric Cancer. Lancet (London England) (2020) 396:635-48. doi: 10.1016/s01406736(20)31288-5

2. Jemal A, Bray F, Center MM, Ferlay J, Ward E, Forman D. Global Cancer Statistics. CA Cancer J Clin (2011) 61:69-90. doi: 10.3322/caac.20107

3. Torre LA, Bray F, Siegel RL, Ferlay J, Lortet-Tieulent J, Jemal A. Global Cancer Statistics, 2012. CA Cancer J Clin (2015) 65:87-108. doi: 10.3322/ caac. 21262

4. Maecker HT, Todd SC, Levy S. The Tetraspanin Superfamily: Molecular Facilitators. FASEB J (1997) 11:428-42. doi: 10.1007/s007920050022

5. Stipp CS, Kolesnikova TV, Hemler ME. Functional Domains in Tetraspanin Proteins. Trends Biochem Sci (2003) 28:106-12. doi: 10.1016/s0968-0004 (02)00014-2

6. Charrin S, le Naour F, Silvie O, Milhiet PE, Boucheix C, Rubinstein E. Lateral Organization of Membrane Proteins: Tetraspanins Spin Their Web. Biochem J (2009) 420:133-54. doi: 10.1042/bj20082422

7. The Human Protein Atlas. (2021). Available at: https://www.proteinatlas. org/search/tetraspanin (Accessed May 29, 2021).

8. Hemler ME. Tetraspanin Functions and Associated Microdomains. Nat Rev Mol Cell Biol (2005) 6:801-11. doi: 10.1038/nrm1736

9. Yáñez-Mó M, Barreiro O, Gordon-Alonso M, Sala-Valdés M, SánchezMadrid F. Tetraspanin-Enriched Microdomains: A Functional Unit in Cell Plasma Membranes. Trends Cell Biol (2009) 19:434-46. doi: 10.1016/ j.tcb.2009.06.004

10. Hemler ME. Tetraspanin Proteins Promote Multiple Cancer Stages. Nat Rev Cancer (2014) 14:49-60. doi: 10.1038/nrc3640

11. Kanetaka K, Sakamoto M, Yamamoto Y, Yamasaki S, Lanza F, Kanematsu T, et al. Overexpression of Tetraspanin CO-029 in Hepatocellular Carcinoma. J Hepatol (2001) 35:637-42. doi: 10.1016/s01688278(01)00183-0

12. Gesierich S, Paret C, Hildebrand D, Weitz J, Zgraggen K, SchmitzWinnenthal FH, et al. Colocalization of the Tetraspanins, CO-029 and CD151, With Integrins in Human Pancreatic Adenocarcinoma: Impact on Cell Motility. Clin Cancer Res (2005) 11:2840-52. doi: 10.1158/10780432.ccr-04-1935

13. Greco C, Bralet MP, Ailane N, Dubart-Kupperschmitt A, Rubinstein E, Le Naour F, et al. E-Cadherin/p120-Catenin and Tetraspanin Co-029 Cooperate for Cell Motility Control in Human Colon Carcinoma. Cancer Res (2010) 70:7674-83. doi: 10.1158/0008-5472.can-09-4482

\section{AUTHOR CONTRIBUTIONS}

YD and SC designed and wrote all the sections of the manuscript. JS contributed to the data collection. HP supervised and revised the review. All authors contributed to the article and approved the submitted version.

\section{FUNDING}

This study was conducted with the support by the National Natural Science Foundation of China (Grant Nos. 82073400).

\section{ACKNOWLEDGMENTS}

We would like to thank Jun Zhao for his support and discussion of the manuscript, and Wei Wang for discussion of manuscript preparations.

14. Voglstaetter M, Thomsen AR, Nouvel J, Koch A, Jank P, Navarro EG, et al Tspan8 Is Expressed in Breast Cancer and Regulates E-Cadherin/Catenin Signalling and Metastasis Accompanied by Increased Circulating Extracellular Vesicles. J Pathol (2019) 248:421-37. doi: 10.1002/path.5281

15. Zoller M. Tetraspanins: Push and Pull in Suppressing and Promoting Metastasis. Nat Rev Cancer (2009) 9:40-55. doi: 10.1038/nrc2543

16. Bonnet M, Maisonial-Besset A, Zhu Y, Witkowski T, Roche G, Boucheix C, et al. Targeting the Tetraspanins With Monoclonal Antibodies in Oncology: Focus on TSPAN8/Co-029. Cancers (2019) 11:179. doi: 10.3390/ cancers11020179

17. Gesierich S, Berezovskiy I, Ryschich E, Zoller M. Systemic Induction of the Angiogenesis Switch by the Tetraspanin D6.1A/CO-029. Cancer Res (2006) 66:7083-94. doi: 10.1158/0008-5472.can-06-0391

18. Claas C, Seiter S, Claas A, Savelyeva L, Schwab M, Zoller M. Association Between the Rat Homologue of CO-029, A Metastasis-Associated Tetraspanin Molecule and Consumption Coagulopathy. J Cell Biol (1998) 141:267-80. doi: 10.1083/jcb.141.1.267

19. Yue S, Zhao K, Erb U, Rana S, Zöller M. Joint Features and Complementarities of TSPAN8 and CD151 Revealed in Knockdown and Knockout Models. Biochem Soc Trans (2017) 45:437-47. doi: 10.1042/ bst20160298

20. Andreu Z, Yanez-Mo M. Tetraspanins in Extracellular Vesicle Formation and Function. Front Immunol (2014) 5:442. doi: 10.3389/fimmu.2014.00442

21. Matsumura N, Zembutsu H, Yamaguchi K, Sasaki K, Tsuruma T, Nishidate $\mathrm{T}$, et al. Identification of Novel Molecular Markers for Detection of Gastric Cancer Cells in the Peripheral Blood Circulation Using Genome-Wide Microarray Analysis. Exp Ther Med (2011) 2:705-13. doi: 10.3892/ etm. 2011.252

22. Mottaghi-Dastjerdi N, Soltany-Rezaee-Rad M, Sepehrizadeh Z, Roshandel G, Ebrahimifard F, Setayesh N. Identification of Novel Genes Involved in Gastric Carcinogenesis by Suppression Subtractive Hybridization. Hum Exp Toxicol (2015) 34:3-11. doi: 10.1177/0960327114532386

23. Zhu H, Wu Y, Zheng W, Lu S. Co-029 Is Overexpressed in Gastric Cancer and Mediates the Effects of EGF on Gastric Cancer Cell Proliferation and Invasion. Int J Mol Med (2015) 35:798-802. doi: 10.3892/ijmm. 2015.2069

24. Wei L, Li Y, Suo Z. TSPAN8 Promotes Gastric Cancer Growth and Metastasis Via ERK MAPK Pathway. Int J Clin Exp Med (2015) 8:8599-607.

25. Lin H, Zhou AJ, Zhang JY, Liu SF, Gu JX. MiR-324-5p Reduces Viability and Induces Apoptosis in Gastric Cancer Cells Through Modulating TSPAN8. J Pharm Pharmacol (2018) 70:1513-20. doi: 10.1111/jphp.12995 
26. Sincock PM, Mayrhofer G, Ashman LK. Localization of the Transmembrane 4 Superfamily (TM4SF) Member PETA-3 (CD151) in Normal Human Tissues: Comparison With CD9, CD63, and alpha5beta1 Integrin. J Histochem Cytochem (1997) 45:515-25. doi: 10.1177/002215549704500404

27. Wang HX, Li Q, Sharma C, Knoblich K, Hemler ME. Tetraspanin Protein Contributions to Cancer. Biochem Soc Trans (2011) 39:547-52. doi: 10.1042/ bst0390547

28. Karamatic Crew V, Burton N, Kagan A, Green CA, Levene C, Flinter F, et al. CD151, the First Member of the Tetraspanin (TM4) Superfamily Detected on Erythrocytes, Is Essential for the Correct Assembly of Human Basement Membranes in Kidney and Skin. Blood (2004) 104:2217-23. doi: 10.1182/ blood-2004-04-1512

29. Ke AW, Shi GM, Zhou J, Wu FZ, Ding ZB, Hu MY, et al. Role of Overexpression of CD151 and/or c-Met in Predicting Prognosis of Hepatocellular Carcinoma. Hepatol (Baltimore Md) (2009) 49:491-503. doi: $10.1002 /$ hep.22639

30. Yang YM, Zhang ZW, Liu QM, Sun YF, Yu JR, Xu WX. Overexpression of CD151 Predicts Prognosis in Patients With Resected Gastric Cancer. PloS One (2013) 8:e58990. doi: 10.1371/journal.pone.0058990

31. Zhai R, Kan X, Wang B, Du H, Long Y, Wu H, et al. miR-152 Suppresses Gastric Cancer Cell Proliferation and Motility by Targeting CD151. Tumour Biol (2014) 35:11367-73. doi: 10.1007/s13277-014-2471-2

32. Ding J, Li D, Gong M, Wang J, Huang X, Wu T, et al. Expression and Clinical Significance of the Long Non-Coding RNA PVT1 in Human Gastric Cancer. Onco Targets Ther (2014) 7:1625-30. doi: 10.2147/ott.s68854

33. Li T, Meng XL, Yang WQ. Long Noncoding RNA PVT1 Acts as a "Sponge" to Inhibit microRNA-152 in Gastric Cancer Cells. Dig Dis Sci (2017) 62:3021-8. doi: 10.1007/s10620-017-4508-z

34. Serru V, Dessen P, Boucheix C, Rubinstein E. Sequence and Expression of Seven New Tetraspans. Biochim Biophys Acta (2000) 1478:159-63. doi: 10.1016/s0167-4838(00)00022-4

35. Chen L, Li X, Wang GL, Wang Y, Zhu YY, Zhu J. Clinicopathological Significance of Overexpression of TSPAN1, Ki67 and CD34 in Gastric Carcinoma. Tumori (2008) 94:531-8. doi: 10.1177/030089160809400415

36. Lu Z, Luo T, Nie M, Pang T, Zhang X, Shen X, et al. TSPAN1 Functions as an Oncogene in Gastric Cancer and Is Downregulated by miR-573. FEBS Lett (2015) 589:1988-94. doi: 10.1016/j.febslet.2015.05.044

37. Qi W, Sun L, Liu N, Zhao S, Lv J, Qiu W. Tetraspanin Family Identified as the Central Genes Detected in Gastric Cancer Using Bioinformatics Analysis. Mol Med Rep (2018) 18:3599-610. doi: 10.3892/mmr. 2018.9360

38. Andria ML, Hsieh CL, Oren R, Francke U, Levy S. Genomic Organization and Chromosomal Localization of the TAPA-1 Gene. J Immunol (Baltimore Md 1950) (1991) 147:1030-6. doi: 10.1515/crll.1993.442.91

39. Oren R, Takahashi S, Doss C, Levy R, Levy S. TAPA-1, the Target of an Antiproliferative Antibody, Defines a New Family of Transmembrane Proteins. Mol Cell Biol (1990) 10:4007-15. doi: 10.1128/mcb.10.8.4007

40. Levy S, Todd SC, Maecker HT. CD81 (TAPA-1): A Molecule Involved in Signal Transduction and Cell Adhesion in the Immune System. Annu Rev Immunol (1998) 16:89-109. doi: 10.1146/annurev.immunol.16.1.89

41. Tedder TF, Inaoki M, Sato S. The CD19-CD21 Complex Regulates Signal Transduction Thresholds Governing Humoral Immunity and Autoimmunity. Immunity (1997) 6:107-18. doi: 10.1016/s1074-7613(00) 80418-5

42. Todd SC, Lipps SG, Crisa L, Salomon DR, Tsoukas CD. CD81 Expressed on Human Thymocytes Mediates Integrin Activation and Interleukin 2Dependent Proliferation. J Exp Med (1996) 184:2055-60. doi: 10.1084/ jem.184.5.2055

43. Rubinstein E, Ziyyat A, Prenant M, Wrobel E, Wolf JP, Levy S, et al. Reduced Fertility of Female Mice Lacking CD81. Dev Biol (2006) 290:351-8. doi: 10.1016/j.ydbio.2005.11.031

44. Charrin S, Latil M, Soave S, Polesskaya A, Chretien F, Boucheix C, et al. Normal Muscle Regeneration Requires Tight Control of Muscle Cell Fusion by Tetraspanins CD9 and CD81. Nat Commun (2013) 4:1674. doi: 10.1038/ ncomms 2675

45. Pileri P, Uematsu Y, Campagnoli S, Galli G, Falugi F, Petracca R, et al. Binding of Hepatitis C Virus to CD81. Science (New York NY) (1998) 282:938-41. doi: 10.1126/science.282.5390.938
46. Luo RF, Zhao S, Tibshirani R, Myklebust JH, Sanyal M, Fernandez R, et al. CD81 Protein Is Expressed at High Levels in Normal Germinal Center B Cells and in Subtypes of Human Lymphomas. Hum Pathol (2010) 41:27180. doi: 10.1016/j.humpath.2009.07.022

47. Muzzafar T, Medeiros LJ, Wang SA, Brahmandam A, Thomas DA, Jorgensen JL. Aberrant Underexpression of CD81 in Precursor B-Cell Acute Lymphoblastic Leukemia: Utility in Detection of Minimal Residual Disease by Flow Cytometry. Am J Clin Pathol (2009) 132:692-8. doi: 10.1309/ajcp02rpvoktnwec

48. Yoo TH, Ryu BK, Lee MG, Chi SG. CD81 Is a Candidate Tumor Suppressor Gene in Human Gastric Cancer. Cell Oncol (Dordr) (2013) 36:141-53. doi: $10.1007 / \mathrm{s} 13402-012-0119-\mathrm{z}$

49. Xu L, Hou Y, Tu G, Chen Y, Du YE, Zhang H, et al. Nuclear Drosha Enhances Cell Invasion Via an EGFR-ERK1/2-MMP7 Signaling Pathway Induced by Dysregulated miRNA-622/197 and Their Targets LAMC2 and CD82 in Gastric Cancer. Cell Death Dis (2017) 8:e2642. doi: 10.1038/ cddis. 2017.5

50. Garcia-Frigola C, Burgaya F, Calbet M, de Lecea L, Soriano E. Mouse Tspan5, a Member of the Tetraspanin Superfamily, Is Highly Expressed in Brain Cortical Structures. Neuroreport (2000) 11:3181-5. doi: 10.1097/00001756200009280-00027

51. Iwai K, Ishii M, Ohshima S, Miyatake K, Saeki Y. Expression and Function of Transmembrane-4 Superfamily (Tetraspanin) Proteins in Osteoclasts: Reciprocal Roles of Tspan-5 and NET-6 During Osteoclastogenesis. Allergol Int (2007) 56:457-63. doi: 10.2332/allergolint. O-07-488

52. He P, Wang S, Zhang X, Gao Y, Li J-L. TSPAN5 Is an Independent Favourable Prognostic Factor and Suppresses Tumour Growth in Gastric Cancer. Oncotarget (2016) 7:40160-73. doi: 10.18632/oncotarget.9514

53. Protty MB, Watkins NA, Colombo D, Thomas SG, Heath VL, Herbert JM, et al. Identification of TSPAN9 as a Novel Platelet Tetraspanin and the Collagen Receptor GPVI as a Component of Tetraspanin Microdomains. Biochem J (2009) 417:391-400. doi: 10.1042/bj20081126

54. Li PY, Lv J, Qi WW, Zhao SF, Sun LB, Liu N, et al. Tspan9 Inhibits the Proliferation, Migration and Invasion of Human Gastric Cancer SGC7901 Cells Via the ERK1/2 Pathway. Oncol Rep (2016) 36:448-54. doi: 10.3892/ or.2016.4805

55. Qi Y, Lv J, Liu S, Sun L, Wang Y, Li H, et al. TSPAN9 and EMILIN1 Synergistically Inhibit the Migration and Invasion of Gastric Cancer Cells by Increasing TSPAN9 Expression. BMC Cancer (2019) 19:630. doi: 10.1186/ s12885-019-5810-2

56. Kong KL, Kwong DL, Fu L, Chan TH, Chen L, Liu H, et al. Characterization of a Candidate Tumor Suppressor Gene Uroplakin 1A in Esophageal Squamous Cell Carcinoma. Cancer Res (2010) 70:8832-41. doi: 10.1158/ 0008-5472.Can-10-0779

57. Zheng Y, Wang DD, Wang W, Pan K, Huang CY, Li YF, et al. Reduced Expression of Uroplakin 1A Is Associated With the Poor Prognosis of Gastric Adenocarcinoma Patients. PloS One (2014) 9:e93073. doi: 10.1371/ journal.pone.0093073

58. Jones NH, Borowitz MJ, Metzgar RS. Characterization and Distribution of a 24,000-Molecular Weight Antigen Defined by a Monoclonal Antibody (DU-ALL-1) Elicited to Common Acute Lymphoblastic Leukemia (cALL) Cells. Leuk Res (1982) 6:449-64. doi: 10.1016/01452126(82)90002-9

59. Morrish DW, Shaw AR, Seehafer J, Bhardwaj D, Paras MT. Preparation of Fibroblast-Free Cytotrophoblast Cultures Utilizing Differential Expression of the CD9 Antigen. In Vitro Cell Dev Biol (1991) 27a:303-6. doi: 10.1007/ bf02630907

60. Miyake M, Koyama M, Seno M, Ikeyama S. Identification of the MotilityRelated Protein (MRP-1), Recognized by Monoclonal Antibody M31-15, Which Inhibits Cell Motility. J Exp Med (1991) 174:1347-54. doi: 10.1084/ jem.174.6.1347

61. Ikeyama S, Koyama M, Yamaoko M, Sasada R, Miyake M. Suppression of Cell Motility and Metastasis by Transfection With Human Motility-Related Protein (MRP-1/CD9) DNA. J Exp Med (1993) 177:1231-7. doi: 10.1084/ jem.177.5.1231

62. Berditchevski F. Complexes of Tetraspanins With Integrins: More Than Meets the Eye. J Cell Sci (2001) 114:4143-51. doi: 10.1038/embor.2012.135 
63. Powner D, Kopp PM, Monkley SJ, Critchley DR, Berditchevski F. Tetraspanin CD9 in Cell Migration. Biochem Soc Trans (2011) 39:563-7. doi: $10.1042 /$ bst0390563

64. Hotta H, Ross AH, Huebner K, Isobe M, Wendeborn S, Chao MV, et al. Molecular Cloning and Characterization of an Antigen Associated With Early Stages of Melanoma Tumor Progression. Cancer Res (1988) 48:2955-62.

65. Atkinson B, Ernst CS, Ghrist BF, Herlyn M, Blaszczyk M, Ross AH, et al. Identification of Melanoma-Associated Antigens Using Fixed Tissue Screening of Antibodies. Cancer Res (1984) 44:2577-81.

66. Barrio MM, Bravo AI, Portela P, Hersey P, Mordoh J. A New Epitope on Human Melanoma-Associated Antigen CD63/ME491 Expressed by Both Primary and Metastatic Melanoma. Hybridoma (1998) 17:355-64. doi: 10.1089/hyb.1998.17.355

67. Chen Z, Gu S, Trojanowicz B, Liu N, Zhu G, Dralle H, et al. DownRegulation of TM4SF Is Associated With the Metastatic Potential of Gastric Carcinoma TM4SF Members in Gastric Carcinoma. World J Surg Oncol (2011) 9:43. doi: 10.1186/1477-7819-9-43

68. Miki Y, Yashiro M, Okuno T, Kitayama K, Masuda G, Hirakawa K, et al. CD9-Positive Exosomes From Cancer-Associated Fibroblasts Stimulate the Migration Ability of Scirrhous-Type Gastric Cancer Cells. Br J Cancer (2018) 118:867-77. doi: 10.1038/bjc.2017.487

69. Gaugitsch HW, Hofer E, Huber NE, Schnabl E, Baumruker T. A New Superfamily of Lymphoid and Melanoma Cell Proteins With Extensive Homology to Schistosoma Mansoni Antigen Sm23. Eur J Immunol (1991) 21:377-83. doi: 10.1002/eji.1830210219

70. Ichikawa T, Ichikawa Y, Isaacs JT. Genetic Factors and Suppression of Metastatic Ability of Prostatic Cancer. Cancer Res (1991) 51:3788-92. doi: 10.1002/10970142(19910715)68:2<451::AID-CNCR2820680241>3.0.CO;2

71. Dong JT, Lamb PW, Rinker-Schaeffer CW, Vukanovic J, Ichikawa T, Isaacs JT, et al. KAI1, A Metastasis Suppressor Gene for Prostate Cancer on Human Chromosome 11p11.2. Science (New York NY) (1995) 268:884-6. doi: $10.1126 /$ science. 7754374

72. Liu WM, Zhang XA. KAI1/CD82, a Tumor Metastasis Suppressor. Cancer Lett (2006) 240:183-94. doi: 10.1016/j.canlet.2005.08.018

73. Tsai YC, Weissman AM. Dissecting the Diverse Functions of the Metastasis Suppressor CD82/KAI1. FEBS Lett (2011) 585:3166-73. doi: 10.1016/ j.febslet.2011.08.031

74. Abe M, Sugiura T, Takahashi M, Ishii K, Shimoda M, Shirasuna K. A Novel Function of CD82/KAI-1 on E-Cadherin-Mediated Homophilic Cellular Adhesion of Cancer Cells. Cancer Lett (2008) 266:163-70. doi: 10.1016/ j.canlet.2008.02.058

75. Hinoda Y, Adachi Y, Takaoka A, Mitsuuchi H, Satoh Y, Itoh F, et al. Decreased Expression of the Metastasis Suppressor Gene KAIl in Gastric Cancer. Cancer Lett (1998) 129:229. doi: 10.1016/S0304-3835(98)00112-8

76. Guan-Zhen Y, Ying C, Can-Rong N, Guo-Dong W, Jian-Xin Q, Jie-Jun W. Reduced Protein Expression of Metastasis-Related Genes (nm23, KISS1, KAI1 and p53) in Lymph Node and Liver Metastases of Gastric Cancer. Int J Exp Pathol (2007) 88:175-83. doi: 10.1111/j.1365-2613.2006.00510.x

77. Zheng H, Tsuneyama K, Cheng C, Takahashi H, Cui Z, Nomoto K, et al. Expression of KAI1 and Tenascin, and Microvessel Density Are Closely Correlated With Liver Metastasis of Gastrointestinal Adenocarcinoma. J Clin Pathol (2007) 60:50-6. doi: 10.1136/jcp.2006.036699

78. Nakamoto T, Murayama Y, Oritani K, Boucheix C, Rubinstein E, Nishida M, et al. A Novel Therapeutic Strategy With Anti-CD9 Antibody in Gastric Cancers. J Gastroenterol (2009) 44:889-96. doi: 10.1007/s00535-009-0081-3

79. Murayama Y, Oritani K, Tsutsui S. Novel CD9-Targeted Therapies in Gastric Cancer. World J Gastroenterol (2015) 21:3206-13. doi: 10.3748/wjg.v21.i11.3206

80. Murayama Y, Miyagawa J, Oritani K, Yoshida H, Yamamoto K, Kishida O, et al. CD9-Mediated Activation of the P46 Shc Isoform Leads to Apoptosis in Cancer Cells. J Cell Sci (2004) 117:3379-88. doi: 10.1242/jcs.01201

81. Liu LX, Liu ZH, Jiang HC, Qu X, Zhang WH, Wu LF, et al. Profiling of Differentially Expressed Genes in Human Gastric Carcinoma by cDNA Expression Array. World J Gastroenterol (2002) 8:580-5. doi: 10.3748/ wjg.v8.i4.580

82. Zhang D, Fan D. Multidrug Resistance in Gastric Cancer: Recent Research Advances and Ongoing Therapeutic Challenges. Expert Rev Anticancer Ther (2007) 7:1369-78. doi: 10.1586/14737140.7.10.1369
83. Cui J, Jiang W, Wang S, Wang L, Xie K. Role of Wnt/beta-catenin Signaling in Drug Resistance of Pancreatic Cancer. Curr Pharm Des (2012) 18:246471. doi: $10.2174 / 13816128112092464$

84. Yang Z, Guo L, Liu D, Sun L, Chen H, Deng Q, et al. Acquisition of Resistance to Trastuzumab in Gastric Cancer Cells Is Associated With Activation of IL-6/STAT3/Jagged-1/Notch Positive Feedback Loop. Oncotarget (2015) 6:5072-87. doi: 10.18632/oncotarget.3241

85. Li L, Yang D, Cui D, Li Y, Nie Z, Wang J, et al. Quantitative Proteomics Analysis of the Role of Tetraspanin-8 in the Drug Resistance of Gastric Cancer. Int J Oncol (2018) 52:473-84. doi: 10.3892/ijo.2017.4231

86. Leelakanok N, Geary S, Salem A. Fabrication and Use of Poly(d,l-lactide-coglycolide)-Based Formulations Designed for Modified Release of 5-Fluorouracil. J Pharm Sci (2018) 107:513-28. doi: 10.1016/j.xphs. 2017.10.012

87. Park JB, Lee JS, Lee MS, Cha EY, Kim S, Sul JY. Corosolic Acid Reduces 5FU Chemoresistance in Human Gastric Cancer Cells by Activating AMPK. Mol Med Rep (2018) 18:2880-8. doi: 10.3892/mmr.2018.9244

88. Qi Y, Qi W, Liu S, Sun L, Ding A, Yu G, et al. TSPAN9 Suppresses the Chemosensitivity of Gastric Cancer to 5-Fluorouracil by Promoting Autophagy. Cancer Cell Int (2020) 20:4. doi: 10.1186/s12935-019-1089-2

89. Zhang Y, Yuan Z, Shen R, Jiang Y, Xu W, Gu M, et al. Identification of Biomarkers Predicting the Chemotherapeutic Outcomes of Capecitabine and Oxaliplatin in Patients With Gastric Cancer. Oncol Lett (2020) 20:290. doi: 10.3892/ol.2020.12153

90. Wang FH, Ma XJ, Xu D, Luo J. UPK1B Promotes the Invasion and Metastasis of Bladder Cancer Via Regulating the Wnt/ $\beta$-Catenin Pathway. Eur Rev Med Pharmacol Sci (2018) 22:5471-80. doi: 10.26355/ eurrev_201809_15807

91. Dai J, Li ZX, Zhang Y, Ma JL, Zhou T, You WC, et al. Whole Genome Messenger RNA Profiling Identifies a Novel Signature to Predict Gastric Cancer Survival. Clin Trans Gastroenterol (2019) 10:e00004. doi: 10.14309/ ctg.0000000000000004

92. Anami K, Oue N, Noguchi T, Sakamoto N, Sentani K, Hayashi T, et al. TSPAN8, Identified by Escherichia Coli Ampicillin Secretion Trap, Is Associated With Cell Growth and Invasion in Gastric Cancer. Gastric Cancer (2016) 19:370-80. doi: 10.1007/s10120-015-0478-z

93. Hori H, Yano S, Koufuji K, Takeda J, Shirouzu K. CD9 Expression in Gastric Cancer and its Significance. J Surg Res (2004) 117:208-15. doi: 10.1016/ j.jss.2004.01.014

94. Ha SY, Do IG, Lee J, Park SH, Park JO, Kang WK, et al. CD151 Overexpression Is Associated With Poor Prognosis in Patients With pT3 Gastric Cancer. Ann Surg Oncol (2014) 21:1099-106. doi: 10.1245/s10434013-3339-1

95. Kang BW, Lee D, Chung HY, Han JH, Kim YB. Tetraspanin CD151 Expression Associated With Prognosis for Patients With Advanced Gastric Cancer. J Cancer Res Clin Oncol (2013) 139:1835-43. doi: 10.1007/ s00432-013-1503-4

96. Zeng P, Wang YH, Si M, Gu JH, Li P. Tetraspanin CD151 as an Emerging Potential Poor Prognostic Factor Across Solid Tumors: A Systematic Review and Metaanalysis. Oncotarget (2015) 8:5592-602. doi: 10.18632/ oncotarget. 13532

97. Ilhan O, Celik SY, Han U, Onal B. Use of KAI-1 as a Prognostic Factor in Gastric Carcinoma. Eur J Gastroenterol Hepatol (2009) 21:1369-72. doi: 10.1097/MEG.0b013e328323aac9

98. Lu G, Zhou L, Zhang X, Zhu B, Wu S, Song W, et al. The Expression of Metastasis-Associated in Colon Cancer-1 and KAI1 in Gastric Adenocarcinoma and Their Clinical Significance. World J Surg Oncol (2016) 14:276. doi: 10.1186/s12957-016-1033-z

99. Knoener M, Krech T, Puls F, Lehmann U, Kreipe H, Christgen M. Limited Value of KAI1/CD82 Protein Expression as a Prognostic Marker in Human Gastric Cancer. Dis Markers (2012) 32:337-42. doi: 10.3233/DMA-20120896

100. Lee HS, Lee HK, Kim HS, Yang HK, Kim WH. Tumour Suppressor Gene Expression Correlates With Gastric Cancer Prognosis. J Pathol (2003) 200:39-46. doi: 10.1002/path.1288

101. Feng T, Sun L, Qi W, Pan F, Lv J, Guo J, et al. Prognostic Significance of Tspan9 in Gastric Cancer. Mol Clin Oncol (2016) 5:231-6. doi: 10.3892/ mco.2016.961 
102. Su J, Zhang Y, Su H, Zhang C, Li W. A Recurrence Model for Laryngeal Cancer Based on SVM and Gene Function Clustering. Acta Otolaryngol (2017) 137:557-62. doi: 10.1080/00016489.2016.1247984

Conflict of Interest: The authors declare that the research was conducted in the absence of any commercial or financial relationships that could be construed as a potential conflict of interest.
Copyright (c) 2021 Deng, Cai, Shen and Peng. This is an open-access article distributed under the terms of the Creative Commons Attribution License (CC BY). The use, distribution or reproduction in other forums is permitted, provided the original author(s) and the copyright owner(s) are credited and that the original publication in this journal is cited, in accordance with accepted academic practice. No use, distribution or reproduction is permitted which does not comply with these terms. 